\title{
Asma infantil severa
}

\section{Severe asthma in childhood}

\author{
Milena VilLAmIL-Osorio, MD.(1); SONIA M. Restrepo-Gualteros, MD.(2); \\ CARLOS E. RodRíGUEZ-MARTíNEZ, MD. ${ }^{(3)}$
}

\section{Resumen}

El asma es una enfermedad crónica de distribución global heterogénea con importantes diferencias en niños y adultos, incluye todos los grupos étnicos y tiene un impacto socioeconómico considerable, la forma severa afecta al 4,5\% de la población infantil. Se describe el caso de una paciente con asma severa y se revisa la literatura.

Se ha intentado establecer fenotipos de asma severa en niños considerando 4 grupos, de inicio tardío con función pulmonar normal, de inicio temprano, atópico con función pulmonar normal, de inicio temprano atópico con leve limitación al flujo aéreo y de inicio temprano con avanzada limitación del flujo aéreo. El tratamiento incluye manejo farmacológico y no farmacológico y es fundamental la adherencia a las medidas ordenadas.

Palabras clave: asma severa, asma severa en niños, fenotipos, terapia combinada en asma.

\begin{abstract}
Asthma is a chronic diseased characterized by an heterogeneous worldwide distribution and important differences between children and adults. It includes all ethnic groups and has considerable socioeconomic impact. The severe form affects $4,5 \%$ of the pediatric population. We describe the case of a patient with severe asthma and review the literature.

The attempt has been made to describe the phenotypes of severe asthma in children considering 4 groups: of late initiation with normal pulmonary function, of early initiation, atopic with normal pulmonary function, of early atopic initiation with slight airflow limitation, and of early initiation with advanced airflow limitation. Treatment includes pharmacologic and nonpharmacologic management, and adherence to ordered measures is fundamental.
\end{abstract} asthma.

Keywords: severe asthma, severe asthma in children, phenotypes, combined therapy in

\footnotetext{
${ }^{(1)}$ Neumóloga Pediatra Hospital de la Misericordia, Universidad El Bosque. Bogotá, Colombia.

${ }^{(2)}$ Neumóloga Pediatra, Universidad $\mathrm{Na}-$ cional de Colombia, Universidad El Bosque, Hospital de la Misericordia. Bogotá, Colombia.

(3)Neumólogo Pediatra, Universidad Nacional de Colombia, Universidad El Bosque, Universidad Militar Nueva Granada, Bogotá, Colombia. Bogotá, Colombia. Correspondencia: Milena Villamil-Osorio, correo electrónico: mile_villamil1002@ hotmail.com

Recibido: 17/05/15. Aceptado: 27/05/15.
} 


\section{Introducción}

El asma en una enfermedad crónica, de distribución global, que afecta alrededor de 300 millones de personas, cerca al 1 al $8 \%$ de la población, incluye todas las edades y grupos étnicos y tiene un impacto socioeconómico considerable $(1,2)$.

Se ha descrito que la forma severa afecta al $0,5 \%$ de la población general y al $4,5 \%$ de la infantil (3), con variaciones entre las series de acuerdo con la definición aplicada. En 2009, la Organización Mundial de la Salud (OMS) propuso una definición uniforme de asma severa en la que incluyó la severidad intrínseca de la misma, el grado de control y la respuesta a los medicamentos. Se expone el caso de una paciente con asma severa y se hace una revisión de la literatura.

\section{Caso}

Paciente de 4 años y 5 meses de edad, de sexo femenino, quien ingresó a urgencias del Hospital de la Misericordia por cuadro clínico de 5 días de evolución de tos con movilización de secreciones, no emetizante ni cianozante, fiebre de $38^{\circ} \mathrm{C}$, dificultad respiratoria dada por taquipnea y retracciones generalizadas en el tórax, desaturación de oxígeno y broncoosbtrucción. Se inició manejo de la vía aérea.

Es fruto de la segunda gestación, nacimiento pretérmino a las 32 semanas por ruptura prematura de membranas; peso: 2.300 gramos, talla: $45 \mathrm{~cm}$, estancia en Unidad de Cuidado Intensivo Neonatal 11 días, con ventilación mecánica invasiva durante 3 días; egresó en ese entonces sin oxígeno suplementario.

Presentó alergia a la proteína de la leche de vaca y fue hospitalizada al mes de vida durante 8 días. A los 10 meses de edad fue hospitalizada por pielonefritis. Desde los 2 años y medio refirió varias hospitalizaciones por crisis asmática, una de en cuidado intermedio y otra en cuidado intensivo, sin necesidad de ventilación mecánica. Antecedente familiar de hermano con asma severa, compromiso pulmonar por citomegalovirus en tratamiento y linfocitos T CD4 bajos.

Recibía manejo con beclometasona inhalada a dosis moderadas sin respuesta; presentaba tos persistente todos los días del mes.

Se inició manejo con terapia combinada salmeterol más fluticasona 100/500 mcg cada 12 horas. En la tomografía axial computarizada se evidenció engrosamiento del intersticio peribroncovascular con opacidades parenquimatosas en ambos campos pulmonares por atelectasias subsegmetarias. En el lóbulo inferior derecho zonas de opacidad parenquimatosa del espacio aéreo y en vidrio esmerilado, en parches, más compatible con proceso bronconeumónico (figuras 1 y 2).

Por severidad del cuadro, se llevó a broncoscopia flexible con estudios en lavado broncoalveolar (figura 3) y para inmunodeficiencia; los resultados se listan en la tabla 1.

Se evidenció asma de predominio alérgico por inmunoglobulina E elevada; evolucionó a la mejoría, se trató infección por Haemophilus influenzae y se descartó infección por micobacterias, CMV y hongos. Por el diagnoóstico de asma severa se continuó manejo con inhaloterapia combinada. Dada su edad, no se consideró como candidata a tratamiento con terapia inmunomoduladora.

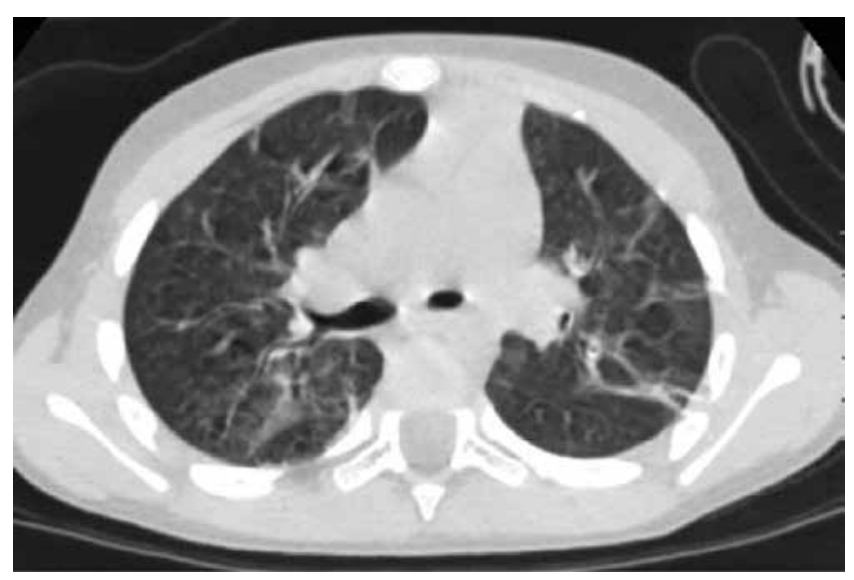

Figura 1. Tomografía axial computarizada con engrosamiento del intersticio peribroncovascular y atelectasias subsegmentarias. 


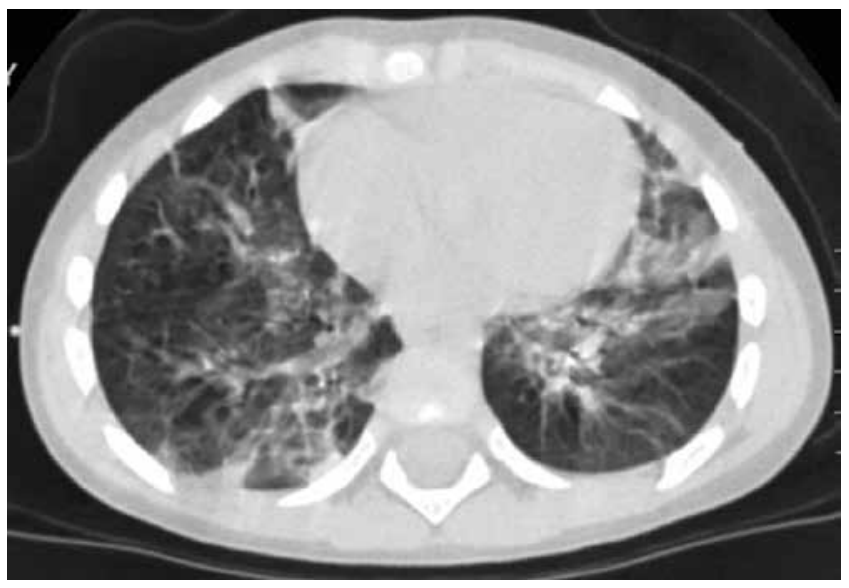

Figura 2. Tomografía axial computarizada con opacidades parenquimatosas en ambos campos pulmonares y atelectasias subsegmetarias. Zonas de opacidad parenquimatosa del espacio aéreo y en vidrio esmerilado, en parches.

\section{Discusión}

El asma severa es una enfermedad heterogénea con peor curso clínico y mayor compromiso de la función pulmonar que el asma persistente no severa (4). En 2009 se intentó unificar la definición y posteriormente en 2014 la European Respiratory Society (ERS) y la American Thoracic Society (ATS) publicaron la guía para asma severa (5); la definición acordada en este consenso se anexa en la tabla 2.

La paciente del caso cumple varios criterios para asma severa: asma no controlada según las Guía GINA, más de dos ciclos de corticoides orales en el último año, ingreso a cuidado intensivo en el último año y dado que no se ha logrado instaurar un manejo médico adecuado con los pasos 4 o 5, según el GINA se desconoce su respuesta pero su curso es severo, criterio incluido por la OMS.

Al asma severa se han asociado varias características. Se considera significativo el antecedente de sensibilización a alergenos con OR 4,73 (IC95\% 1,12-18,53; $\mathrm{p}=0.03$ ), tiempo de hospitalización por asma con OR 3,71 (IC95\% 1,11-12,33; $\mathrm{p}=0.03$ ), visitas a urgencias por asma en el año anterior con $\mathrm{OR}$ $11,98$ (IC95\% 2,7-53,11; $\mathrm{p}=0,001)$ y síntomas desencadenados por actividad física con OR 12,78 (IC $95 \% 2,66-61,4 ; \mathrm{p}=0,001)(4)$.

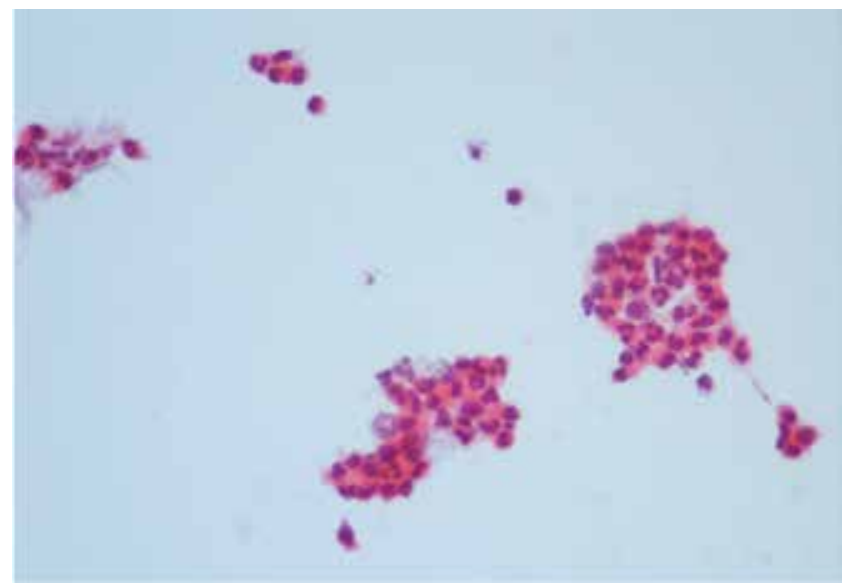

Figura 3. Muestra de lavado broncoalveolar, tinción hematoxilina \& eosina, aumento $40 x$. Predominio de polimorfonucleares neutrófilos y algunos histiocitos; no se observaron microorganismos.

\section{Fenotipos de asma severa en niños}

Se ha intentado establecer fenotipos de asma severa en niños pues estos son diferentes a los descritos en adultos (6), de ahí que los enfoques terapéuticos que parten de los fenotipos en adultos no se pueden extrapolar a los niños. El estudio SARP realizado entre 2001 y 2011 (7), caracterizó cuatro grupos:

1. Inicio tardío con función pulmonar normal.

2. Inicio temprano, atópico con función pulmonar normal.

3. Inicio temprano, atópico con leve limitación al flujo aéreo.

4. Inicio temprano con avanzada limitación del flujo aéreo.

La paciente corresponde inicio temprano y es atópica, tuvo alergia a la proteína de la leche de vaca e inmunoglobulina $\mathrm{E}$ elevada; quedó pendiente el reporte de la función pulmonar.

Dada la heterogeneidad, además de los fenotipos por estudios de grupos, se han estudiado fenotipos por análisis molecular comparando especialmente niños con asma severa y no severa y además 
Tabla 1. Resumen de los principales resultados de los estudios realizados.

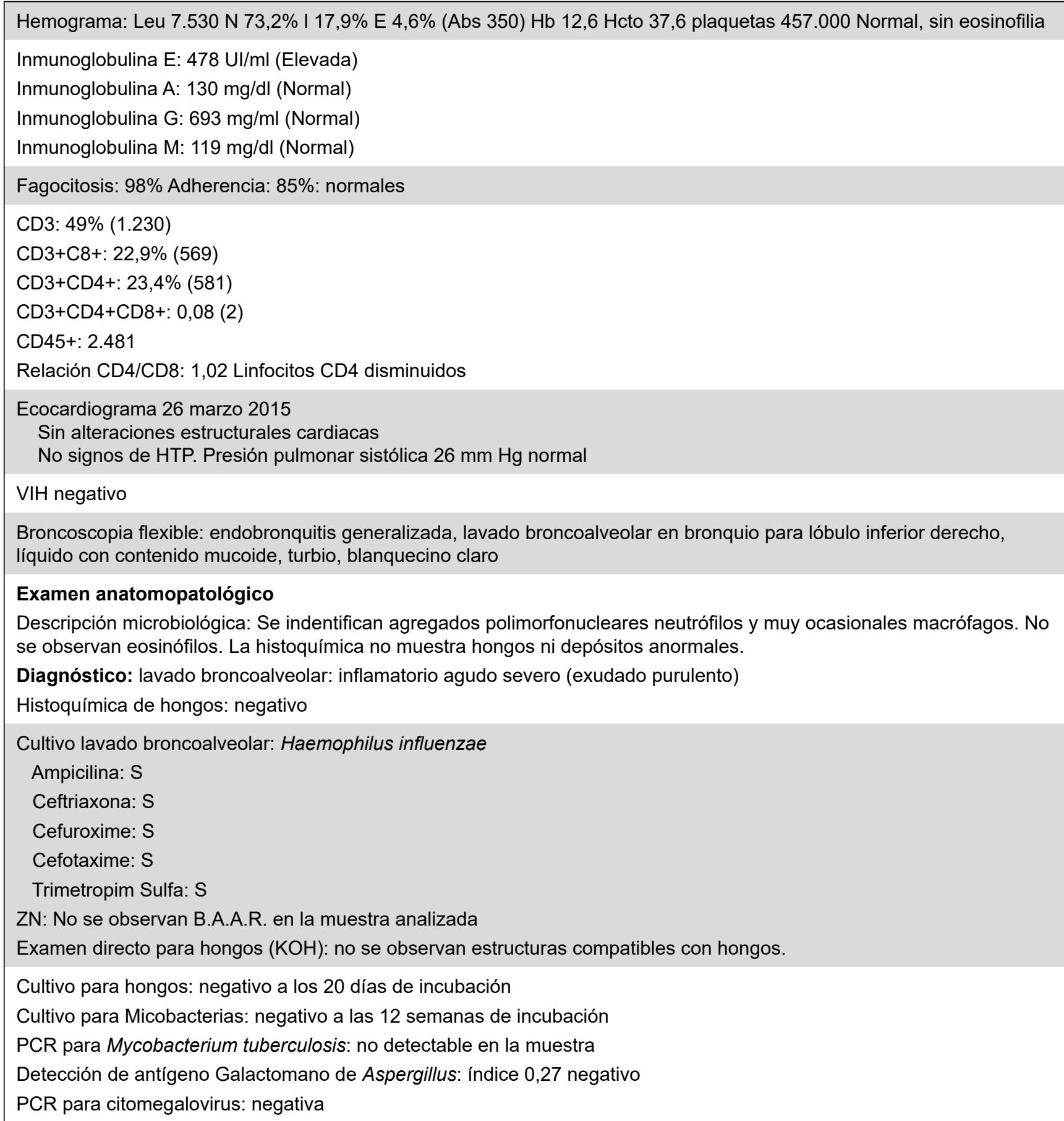

fenotipos basados en genética, con resultados limitados que plantean una respuesta alterada a los corticoides en esta población (8).

Los fenotipos solos no identifican la inmunopatología que permitiría identificar y distinguir la población específica para cada tratamiento; cuando se identifican las características biológicas los fenotipos deben evolucionar a endotipos. De otra parte, integrar genética, biología y clínica debe mejorar la capacidad de tratar con eficacia el asma 
Tabla 2. Definición de asma severa para pacientes menores a 6 años de edad.

Asma que requiere tratamiento con medicamentos incluidos en el paso 4 o 5 según las Guías GINA (altas dosis de corticoides inhalados más Beta 2 agonistas de larga acción o modificadores de leucotrienos/teofilina) en el año previo o corticoides sistémicos por más del $50 \%$ del año previo para prevenir estar "no controlado" o que permanece no controlado a pesar de esta terapia.

Asma no controlada definida como mínimo uno de los siguientes:

1. Pobre control de síntomas: $A C Q$ consistentemente $>1,5, A C T<20$ (O "no bien controlada" por guías NAEPP/GINA).

2. Exacerbaciones frecuentes severas: 2 o más ciclos de corticoides orales ( $>3$ días cada uno) en el año previo.

3. Exacerbación severa: como mínimo una hospitalización, estancia en Unidad de Cuidado Intensivo o ventilación mecánica en el año previo.

4. Limitación al flujo aéreo: tener $\mathrm{VEF}_{1}$ menor al $80 \%$ después de adecuado manejo broncodilatador (teniendo disminuido el VEF ${ }_{1} / C V F$, es decir menor al límite inferior de la normalidad).

Asma controlada que empeora al disminuir estas altas dosis de corticoides inhalados o sistémicos (o biológicos adicionales).

severa en niños (9). Se han descrito, en adultos, endotipos como:

- Alergia de inicio temprano: sensibilidad a aeroalergenos u otra enfermedad alérgica con claros biomarcadores como FeNo, IgE específica y periostina.

- Inicio adulto, asma severa con eosinofilia persistente de progresión no conocida; se han identificado alteraciones en la vía de los cisteinil leucotrienos.

- Micosis broncopulmonares alérgicas con IgE e IgG elevadas para las que entre otros manejos, se aconseja el tratamiento antifúngico.

- Mujer - obesidad con pobre respuesta a corticoides, genética no conocida.

- Neutrofílica con posible respuesta a macrólidos.

Estudios clínicos de manejo del asma guiado con eosinófilos en esputo, han mostrado buenos resultados en adultos pero no han reducido las exacerbaciones ni han mejorado el control del asma en niños (10). Se ha visto que esta población puede tener conteo de eosinófilos fluctuante entre eosinofílicos y no eosinofílicos y se ha cuestionado la presencia del fenotipo neutrofílico, pues aunque se ha observado un aumento de los neutrófilos en la vía aérea de niños con asma severa la cantidad no difiere de niños con asma más leve (11); estudios como el SARP han mostrado potenciales endotipos en niños pero estos no han sido completamente elucidados (12).

El lavado broncoalveolar de la paciente del caso mostró predominio de neutrófilos con un claro desencadenante infeccioso, pero no fue posible definir que este fuera su patrón predominante. Es llamativo, además, el conteo de linfocitos CD4 bajos, patrón que se presenta igualmente en su hermano que tiene diagnóstico de asma severa.

\section{Linfocitos $C D 4 / C D 8^{+}$en asma severa}

El asma severa se caracteriza por un fenotipo molecular de inflamación de la vía aérea diferente que no tiene un claro patrón TH1 o TH2 (13). Variantes genéticas en numerosos genes pueden interactuar e influir en la función pulmonar, la susceptibilidad para el asma y su severidad (14), al igual que la farmacogenética puede condicionar la respuesta al tratamiento. Los cambios epigenéticos pueden llevar a no codificar cambios estructurales en el ADN $y$ efectos de pequeños ARN no codificantes o microARN. Estos últimos regulan la función de las células $\mathrm{T}$ ayudadoras (TH2) llevando a enfermedad alérgica de la vía aérea y producción de interleucina 13; igualmente, se han propuesto microARN en células CD4 y CD8 sin conocer su importancia en asma severa (5). 
Estudios con transcriptómica en sangre periférica, sugieren activación diferencial de células T CD8 ${ }^{+}$ en asma severa, comparado con células $\mathrm{T} \mathrm{CD}^{+}{ }^{+}$secundario a múltiples vías de activación y regulación de la función de las células T $\mathrm{CD} 8^{+}$, dándoles así un lugar importante en la fisiopatología (15).

\section{Tratamiento}

Incluye manejo farmacológico y no farmacológico, y es fundamental la adherencia a las medidas ordenadas (16); la paciente se ha tratado con inhaloterapia con cortoide más beta 2 inhalado de larga acción y no ha tenido recurrencia de exacerbaciones. Así mismo, deben estudiarse y tratarse comorbilidades que puedan descompensar el asma.

\section{Conclusión}

El asma severa es una enfermedad heterogénea con importantes diferencias en niños y adultos, de ahí que sea fundamental el diagnóstico adecuado y enfoque terapéutico individualizado que se facilita al entender los factores que caracterizan y regulan su presentación. Aumentar nuestro entendimiento en fenotipos y endotipos del asma severa en niños podrá llevarnos a desarrollar terapéuticas específicas para esta compleja patología.

\section{Conflictos de interés}

Los autores declaran no tener conflictos de interés.

\section{Bibliografía}

1. Bousquet J, Mantzouranis E, Cruz AA, Aït-Khaled N, BaenaCagnani CE, Bleecker ER, et al. Uniform definition of asthma severity, control, and exacerbations: document presented for the World Health Organization Consultation on Severe Asthma. J Allergy Clin Immunol. 2010;126(5):926-38.

2. 2015 GINA Report, Global Strategy for Asthma Management and Prevention [Internet]. Global Initiative for Asthma - GINA. [Acceso 2016 Apr 10]. Disponible en: http://ginasthma.org/gina-report-global-strategy-for-asthma-managementand-prevention/

3. Lang A, Carlsen KH, Haaland G, Devulapalli CS, MuntheKaas M, Mowinckel P, et al. Severe asthma in childhood: assessed in 10 year olds in a birth cohort study. Allergy. 2008;63(8):1054-60.
4. Montella S, Baraldi E, Cazzato S, Aralla R, Berardi M, Brunetti LM, et al. Severe asthma features in children: a case-control online survey. Ital J Pediatr. 2016;42(1):9.

5. Chung KF, Wenzel SE, Brozek JL, Bush A, Castro M, Sterk PJ, et al. International ERS/ATS guidelines on definition, evaluation and treatment of severe asthma. Eur Respir J. 2014;43(2):343-73.

6. Fainardi V, Saglani S. The need to differentiate between adults and children when treating severe asthma. Expert Rev Respir Med. 2015;9(4):419-28.

7. Fitzpatrick AM, Teague WG, Meyers DA, Peters SP, Li X, Li $\mathrm{H}$, et al. Heterogeneity of severe asthma in childhood: confirmation by cluster analysis of children in the National Institutes of Health/National Heart, Lung, and Blood Institute Severe Asthma Research Program. J Allergy Clin Immunol. 2011;127(2):382-9.e1-13.

8. Guilbert TW, Bacharier LB, Fitzpatrick AM. Severe asthma in children. J Allergy Clin Immunol Pract. 2014;2(5):489-500.

9. Wenzel S. Severe asthma: from characteristics to phenotypes to endotypes. Clin Exp Allergy J Br Soc Allergy Clin Immunol. 2012;42(5):650-8.

10. Fleming L, Wilson N, Regamey N, Bush A. Use of sputum eosinophil counts to guide management in children with severe asthma. Thorax. 2012;67(3):193-8.

11. Coverstone A, Bacharier LB, Fitzpatrick AM. Severe asthma in school-age children: evaluation and phenotypic advances. Curr Allergy Asthma Rep. 2015;15(5):20.

12. Fitzpatrick AM. Severe asthma in children: lessons learned and future directions. J Allergy Clin Immunol Pract. 2016;4(1):11-9.

13. Fitzpatrick AM, Higgins M, Holguin F, Brown LAS, Teague WG, National Institutes of Health/National Heart, Lung, and Blood Institute's Severe Asthma Research Program. The molecular phenotype of severe asthma in children. J Allergy Clin Immunol. 2010;125(4):851-7.e18.

14. Li X, Howard TD, Moore WC, Ampleford EJ, Li H, Busse $\mathrm{WW}$, et al. Importance of hedgehog interacting protein and other lung function genes in asthma. J Allergy Clin Immunol. 2011;127(6):1457-65.

15. Tsitsiou E, Williams AE, Moschos SA, Patel K, Rossios C, Jiang $\mathrm{X}$, et al. Transcriptome analysis shows activation of circulating $\mathrm{CD}^{+} \mathrm{T}$ cells in patients with severe asthma. J Allergy Clin Immunol. 2012;129(1):95-103.

16. Custovic A, Johnston SL, Pavord I, Gaga M, Fabbri L, Bel $\mathrm{EH}$, et al. EAACI position statement on asthma exacerbations and severe asthma. Allergy. 2013;68(12):1520-31.

Revista Colombiana de Neumología Vol 27 No $3 \mid 2015$ 